\title{
Coexpression modules constructed by weighted gene co-expression network analysis indicate ubiquitin-mediated proteolysis as a potential biomarker of uveal melanoma
}

\author{
MENG YANG $^{1 *}$, QI WAN ${ }^{2 *}$, XIANG HU ${ }^{3-6}$, HAITAO YIN $^{3-6}$, DAWEI HAO $^{3-6}$, CHENGJUN WU $^{3-6}$ and JIANMIN LI ${ }^{1}$ \\ ${ }^{1}$ Department of Dermatology, The Third Affiliated Hospital of Guangxi Medical University, Nanning, Guangxi 530031; \\ ${ }^{2}$ Department of Ophthalmology, The People's Hospital of Leshan, Leshan, Sichuan 614000 ; ${ }^{3}$ Department of Radiotherapy, \\ Xuzhou Central Hospital Affiliated to Nanjing University of Chinese Medicine; ${ }^{4}$ Department of Radiotherapy, Xuzhou \\ Hospital Affiliated to The Medical College of Southeast University; ${ }^{5}$ Department of Radiotherapy, Xuzhou Clinical \\ School; ${ }^{6}$ Department of Radiotherapy, Cancer Institute, Xuzhou Medical College, Xuzhou, Jiangsu 221009, P.R. China
}

Received March 2, 2017; Accepted July 27, 2018

DOI: $10.3892 /$ etm.2018.6945

\begin{abstract}
Uveal melanoma (UM) is a tumor that affects individuals throughout the world. Although gene expression analysis of UM has been performed previously, systemic co-expression analysis for this type of cancer remains lacking. Microarray data of UM samples was obtained from the Genome Expression Omnibus (dataset GSE44295). Co-expression modules were built by weighted gene co-expression network analysis. Functional enrichment analysis was performed on the co-expressed genes from important modules. Seven co-expression modules were constructed from the 5,000 genes gathered from the 58 human UM samples. The number of genes in these modules ranged from 73 to 3,051, with the mean number being 711 . There was a marked difference in interactions among pairwise modules. Functional enrichment analysis demonstrated that module 2 was mainly enriched in pathways associated with the regulation of transcription. Additionally, modules 2-4 were significantly enriched in the ubiquitin mediated proteolysis pathway, suggesting it could serve a critical role in the occurrence and development of UM. The findings of the present study present a framework of co-expressed gene modules for human UM and provide
\end{abstract}

Correspondence to: Professor Jianmin Li, Department of Dermatology, The Third Affiliated Hospital of Guangxi Medical University, 13 Dancun Road, Nanning, Guangxi 530031, P.R. China E-mail: jianminli244@sina.com

Dr Xiang $\mathrm{Hu}$, Department of Radiotherapy, Xuzhou Central Hospital Affiliated to Nanjing University of Chinese Medicine, 199 South Road, Xuzhou, Jiangsu 221009, P.R. China

E-mail: huxiangwt@sina.com

*Contributed equally

Key words: uveal melanoma, co-expression, modules, gene expression, function an improved understanding of these modules at a functional level. Understanding the molecular mechanism and cellular pathways involved in pathogenesis of UM is extremely important for the development of more effective diagnostic and therapeutic strategies.

\section{Introduction}

Uveal melanoma (UM) is the most common type of malignant tumor that occurs in the eyes of adults (1). There are 6-7 cases/million people in America each year $(2,3)$. Research on the gene expression module of UM is rare, which limits the understanding of critical genes associated with the occurrence and development of the disease. Despite the advances in the diagnosis and treatment of UM, the prognosis for patients with UM remains poor. UM frequently metastasizes and due to its hematologic nature, the liver is often the first site of metastatic disease. Approximately $50 \%$ of patients with UM will eventually succumb to their disease and the 5-year survival rate was $84 \%$ among all patients (4). Novel markers for UM are urgently required to improve the clinical management of individuals with the disease and increase their life expectancy.

Previously there have been few studies investigating the expression data of UM gene modules, which has limited the overall understanding of the function of critical genes within UM. Weighted gene co-expression network analysis (WGCNA) is a method frequently used to study biological networks with paired correlations between variables (5). WGCNA is a comprehensive collection of R functions for performing various aspects of weighted correlation network analysis (6). This technique has been widely used to study various biological processes, including cancer, genetics and brain imaging data, where it can help identify candidate biomarkers or therapeutic targets (7). Not only can WGCNA compare differentially expressed genes, but it can also discern the interactions between genes in different co-expression modules (8). WGCNA has been reported to have identified independent predictors of life expectancy for patients with breast cancer (7). WGCNA analysis has also been useful in the 
identification of potential oncogenetic drivers and therapeutic targets for patients with small cell lung cancer (9).

The present study aimed to construct co-expression modules using the gene expression data of patients with UM. Gene Ontology (GO) term and Kyoto Encyclopedia of Genes and Genomes (KEGG) pathway enrichment analyses were performed on the modules constructed, in order to establish the primary functions of the genes within these modules. These findings may be valuable for developing novel therapies to treat UM.

\section{Materials and methods}

Cluster analysis of UM microarray data. The microarray dataset GSE44295 and probe signal values were obtained from the Genome Expression Omnibus (GEO) database (ncbi. nlm.nih.gov/geo/query/acc.cgi?acc=GSE44295). This dataset comprised 58 samples of classic UM. The reference nos. of the UM samples were GSM1082563, GSM1082565-GSM1082566, GSM1082568-GSM1082575, GSM1082577-GSM1082584, GSM1082586-GSM1082596 and GSM1082598-GSM1082625. The sequencing platform used was GPL6883 HumanRef-8 Expression BeadChip (version 3.0; Illumina, Inc., San Diego, CA, USA). Microarray information was transformed into gene expression information by the expression value of probes from the GEO dataset. Microarray annotation information was used to match probes with corresponding genes. Probes with more than one gene were eliminated and the average value was calculated for genes corresponding to more than one probe. Samples with negative values were also eliminated. The threshold value was determined by the number of genes with a different threshold of expression. The WGCNA algorithm (7) was used to evaluate the gene expression value. The flashClust tools package (version 1.1.25; cran.r-project.org/web /packages/fastcluster/) in $\mathrm{R}$ language was used to perform the cluster analysis of the samples with appropriate threshold values.

Construction of co-expression modules for UM. The power value was screened out in the construction of each module using the WGCNA algorithm (7). The gradient method was used to test the independence and the average connectivity degree of different modules with different power values (the power value ranging from 1-20). The appropriate power value was determined when the degree of independence was 0.8 . Once the power value was determined, the module construction proceeded using the WGCNA algorithm. The corresponding gene information for each module was extracted and the minimum number of genes was set as 50 to enable high reliability of the results.

Analysis of the co-expression modules. The interaction of co-expression modules was determined using R language (10) and the WGCNA algorithm. The Heatmap tools package (version 1.1.1; cran.r-project.org/web/packages/heatmap3) was used to analyze the strength of the interactions.

Functional enrichment analysis of the co-expression modules. The constructed modules were arranged by the number of genes they contained and then functional enrichment analysis was performed on the genes in these modules. The corresponding gene information was mapped to the Database for Annotation, Visualization and Integrated Discovery (david. ncifcrf.gov/summary.jsp) (11). GO (12) term and KEGG $(13,14)$ pathway enrichment analyses were then performed on the results. $\mathrm{P} \leq 0.05$ after correction was used as the threshold for significant enrichment. The top five records were extracted if there were more than five significant results.

\section{Results}

Cluster analysis of UM samples. Following the elimination of probes with $>1$ gene, a total of 7,576 gene expression values were obtained. Then, the 5,000 genes with the highest average expression value were extracted. The flashClust tools package was used to perform the cluster analysis on these samples and the results are presented in Fig. 1. The 58 samples were divided into two clusters. A total of 38 samples were included in the first cluster, where sample GSM1082605 had the highest expression value. The second cluster included 20 samples, where sample GSM1082592 had the highest expression value.

Construction of co-expression modules for UM. The expression values of 5,000 genes in 58 samples of UM were used to construct co-expression modules using the WGCNA algorithm. One of the critical parameters was the power value, which mainly affected the independence and the average degree of connectivity within co-expression modules. Firstly, the power value was identified (Fig. 2). When the power value was 6 , the independence degree was $\geq 0.8$; the power value could appropriately assess the scale free topology of the network according to the WGCNA algorithm. Therefore, the power value used to construct the co-expression modules was determined to be 6 .

As a result, seven co-expression modules were constructed (Fig. 3). These modules ranged from large to small by the number of genes they contained. The number of genes in the seven modules was $3,051,529,485,453,213,173$ and 73 . The average number of the genes included in the seven modules was 711 . There were 23 genes that did not belong to any of the seven modules.

Interaction analysis of the co-expression modules. Interactions of the seven co-expression modules were analyzed (Fig. 4). There was a clear difference between the interactions among different modules. The degree of connectivity between critical genes was analyzed in order to better clarify the interactions among the constructed co-expression modules (Fig. 5). With the exception of self-comparison, the strongest connectivity interactions were observed between modules 2 and 3 , along with interactions between modules 2 and 7 ( $\sim 0.8)$.

Functional enrichment analysis of genes in the co-expression modules. GO term and KEGG pathway enrichment analyses were performed on the genes in the seven constructed modules (Tables I and II, respectively). There was a significant difference in the biological processes that different modules were enriched in. Genes in module 1 were mainly enriched in translational initiation (GO:0006413), signal recognition particle-dependent co-translational protein targeting to membranes (GO:0006614) and translation (GO:0006412), while genes in module 2 were 


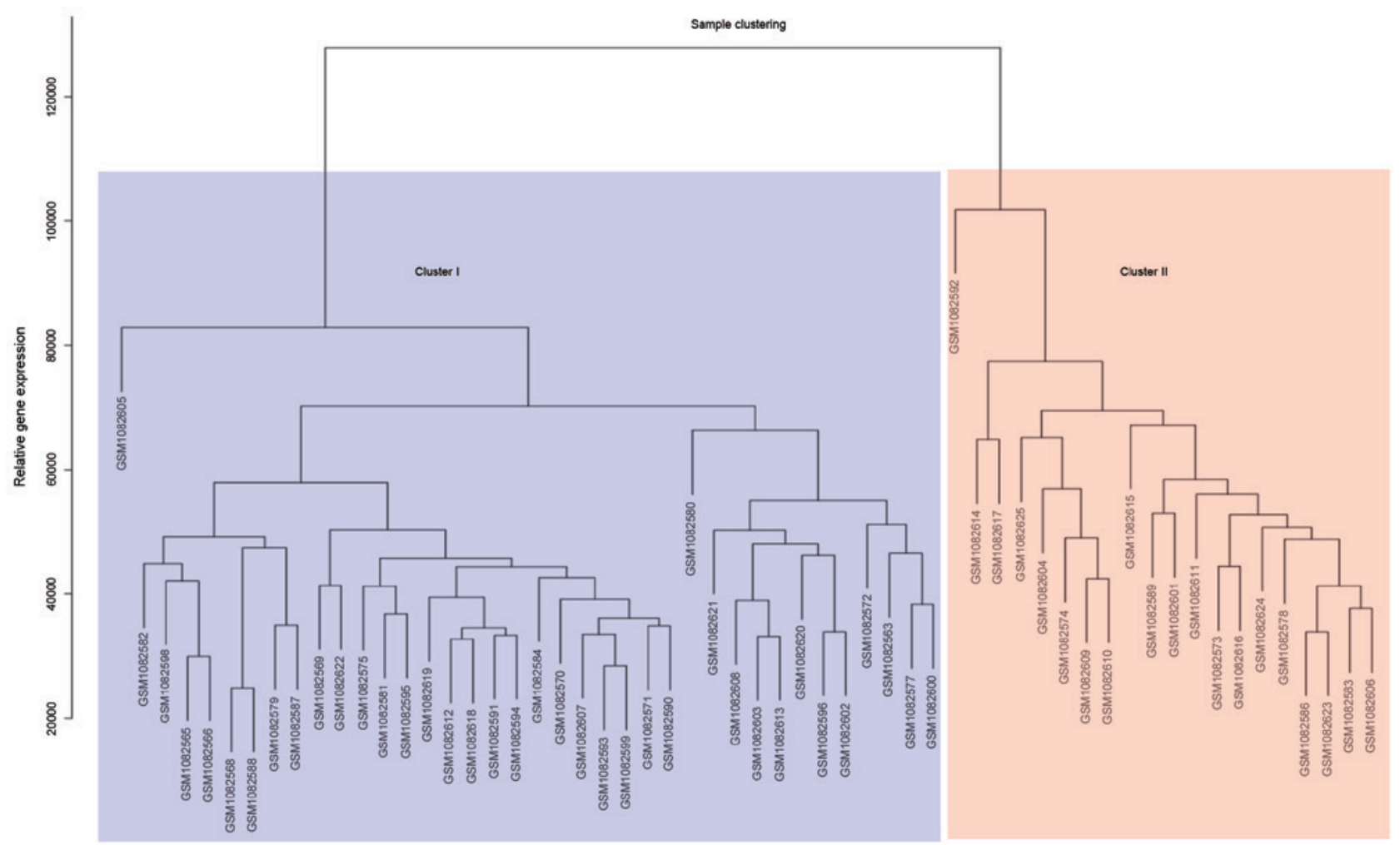

Figure 1. Cluster analysis of uveal melanoma samples. Cluster analysis was performed on the 5,000 genes with the highest average expression values by weighted gene co-expression network analysis and flashClust. All the samples were divided into two clusters, Cluster I (light blue) and Cluster II (light red), which included 38 and 20 samples, respectively.

A

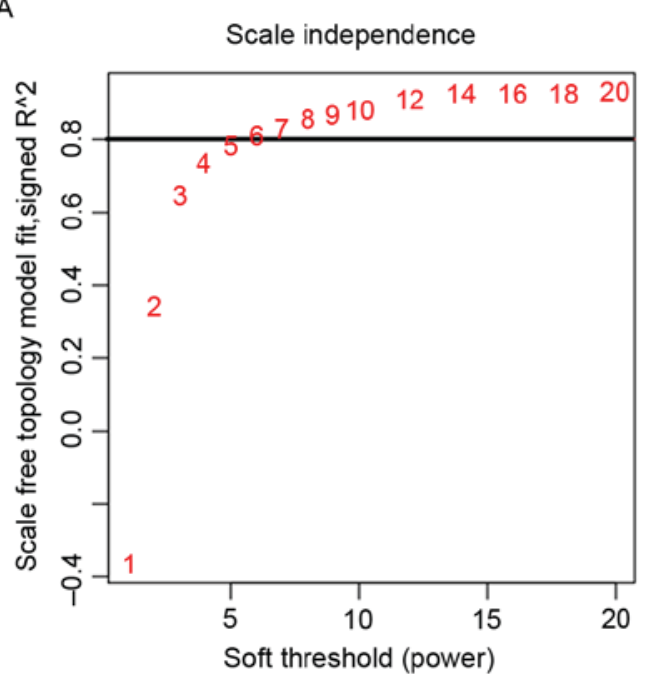

B

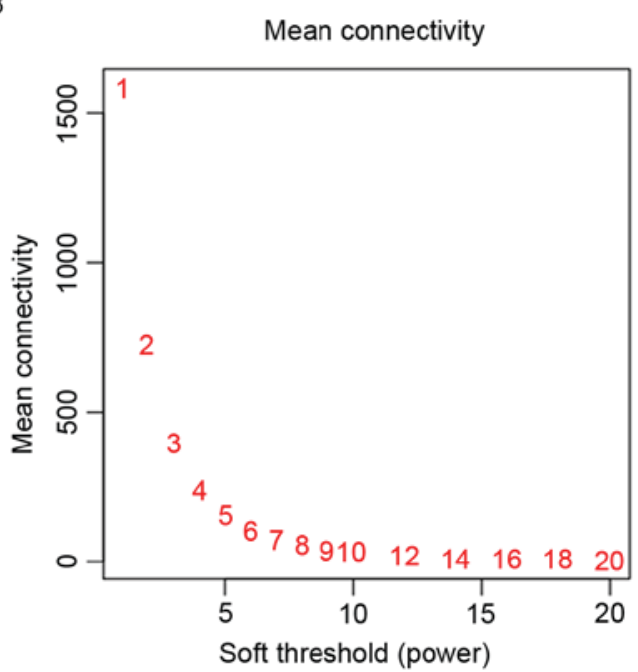

Figure 2. Power value screening of genes in UM co-expression modules. (A) The effect of different power values on the degree of independence between UM co-expression modules. (B) The effect of different power values on the degree of connectivity between UM co-expression modules. UM, uveal melanoma.

mainly enriched in transcriptional regulatory processes, including the regulation of DNA-templated transcription (GO:0006355), DNA-templated transcription (GO:0006351) and transcription from the RNA polymerase II promoter (GO:0006366). Genes in module 3 were mainly enriched in RNA processing and transportation processes, including RNA export from the nucleus (GO:0006405), mRNA splicing via the spliceosome (GO:0000398) and mRNA export from the nucleus (GO:0006406).
Genes in module 4 were similar to that of genes in module 3, which were mainly enriched in mRNA splicing via the spliceosome (GO:0000398). Genes in module 5 were mainly enriched in transcriptional regulation processes, such as the regulation of transcription from RNA polymerase II promoter (GO:0006357). Genes in module 6 were mainly enriched in biological processes associated with RNA processing and transcription, including RNA splicing (GO:0008380) and mRNA export from nucleus 


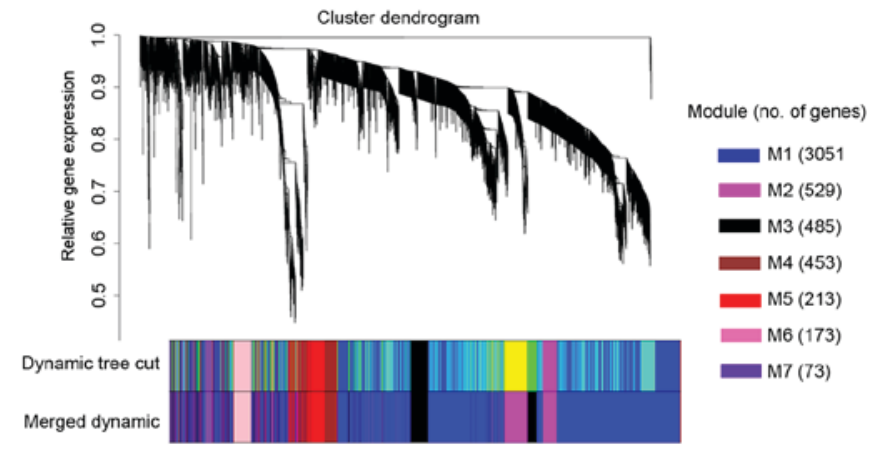

Figure 3. UM co-expression modules constructed. The WGCNA algorithm was used to construct co-expression modules for UM. Each branch represents a gene and the color below indicates each co-expression M. UM, uveal melanoma; WGCNA, weighted gene co-expression network analysis; M, module.

(GO:0006406), which was similar to the result of modules 3 and 4 . Genes in module 7 were mainly enriched in biological processes involving immune responses, including the type I interferon signaling pathway (GO:0060337), the interferon- $\gamma$-mediated signaling pathway (GO:0060333) and immune responses (GO:0006955).

KEGG pathway enrichment analysis was conducted on the genes in the 7 modules (Table II). The results demonstrated that there were significantly enriched pathways within all of the modules except module 5. Genes in module 1 were mainly enriched in metabolic pathways such as the ribosome (hsa03010) and oxidative phosphorylation (hsa00190), while genes in module 2 were mainly enriched in the notch signaling pathway (hsa04330), the RNA degradation pathway (hsa03018), cancer progression pathways (hsa05200) and ubiquitin mediated proteolysis (hsa04120). Genes in module 3 were also mainly enriched in ubiquitin-mediated proteolysis (hsa04120), and pathways associated with RNA transportation and degradation. Genes in module 4 were mainly enriched in ubiquitin-mediated proteolysis (hsa04120), while module 6 was mainly enriched in metabolic pathways associated with RNA transportation and processing. Genes in module 7 were mainly enriched in pathways associated with antigen processing and presentation (hsa04612), and herpes simplex infection (hsa05168).

Module 2 was regarded as a critical module in the occurrence of UM based on the result of the GO and KEGG enrichment analyses. Additionally, the ubiquitin mediated proteolysis (hsa04120) pathway was significantly enriched in modules 2-4. This suggests that ubiquitination may serve an important role in the occurrence and development of UM.

\section{Discussion}

In the present study, a total of seven co-expression modules were constructed from the 5,000 genes from 58 human UM samples using the WGCNA method. The results of the functional enrichment analysis revealed that there was a significant difference in interactions among different modules, and that this was largely associated with their different functions. The results demonstrated that module 2 was enriched in pathways associated with transcriptional regulation processes, and was regarded as a key module

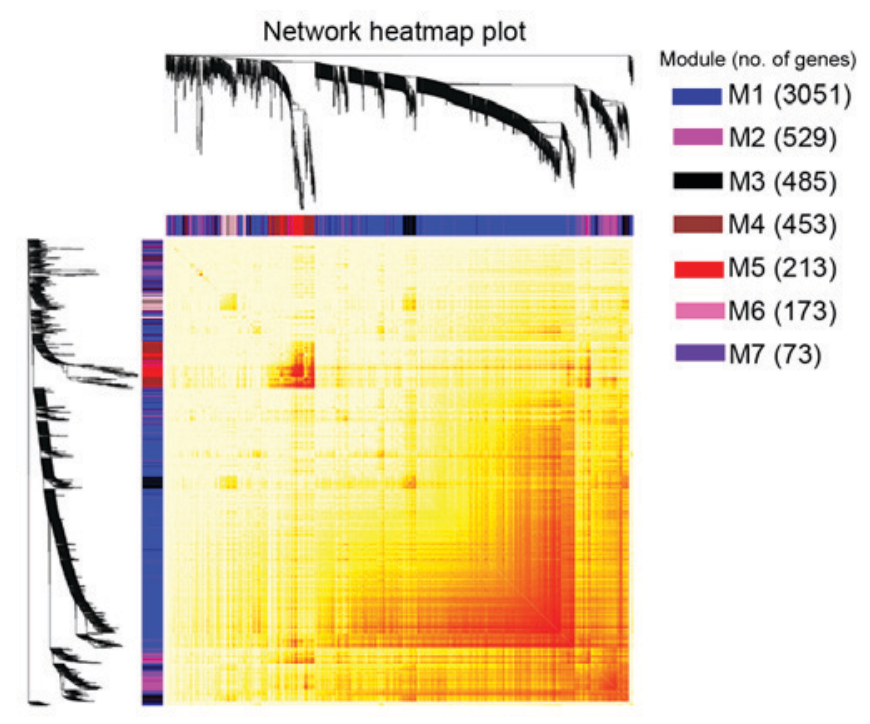

Figure 4. Interactions between genes in the co-expression modules. The different colors on the horizontal and vertical axis represent different modules. The yellow color in the middle represents the relativity among each module. There were notable differences in the correlation among different modules. M, module.

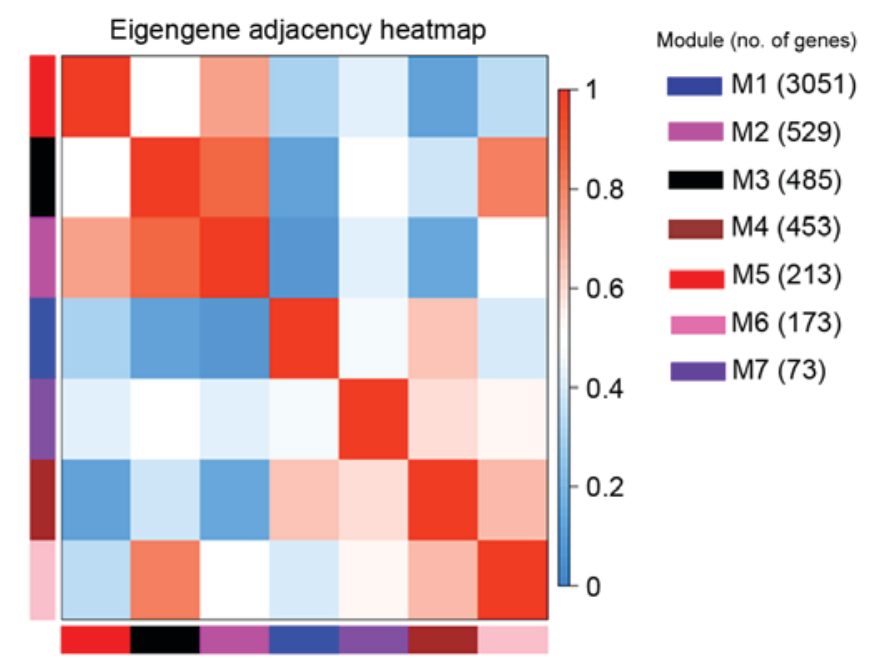

Figure 5. Connectivity analysis of critical genes in different modules. The color from blue (0) to red (1) in the heatmap represents the connectivity of critical genes in different modules from weak to strong, respectively. M, module.

in the occurrence and development of UM. The ubiquitin mediated proteolysis pathway was identified to be significantly enriched in modules $2-4$, suggesting that it may exhibit potential as a prognostic and predictive marker for the survival of patients with UM.

In the present study the WGCNA method was used to construct the seven co-expression modules, each of which included a series of genes with similar expression profiles. Genes within the same module were considered to be associated with each other by function. This method, unlike regular cluster analysis, where clusters are constructed based on the geometric distance of data, was advantageous since the analysis performed by WGCNA has biological significance, making the results more applicable. 
Table I. GO term enrichment analysis of genes in the co-expression modules.

\begin{tabular}{|c|c|c|c|c|c|}
\hline Module & GO no. & Term & $\begin{array}{l}\text { No. of } \\
\text { genes }\end{array}$ & $\begin{array}{l}\text { Background } \\
\text { genes }(\%)\end{array}$ & P-value \\
\hline \multirow[t]{5}{*}{1} & GO:0006413 & Translational initiation & 86 & 2.842975 & $7.33 \mathrm{E}-33$ \\
\hline & GO:0006614 & $\begin{array}{l}\text { SRP-dependent cotranslational protein targeting to } \\
\text { membrane }\end{array}$ & 64 & 2.115702 & $3.31 \mathrm{E}-27$ \\
\hline & GO:0006412 & Translation & 114 & 3.768595 & $2.07 \mathrm{E}-26$ \\
\hline & GO:0000184 & $\begin{array}{l}\text { Nuclear-transcribed mRNA catabolic process, } \\
\text { nonsense-mediated decay }\end{array}$ & 70 & 2.31405 & 2.09E-24 \\
\hline & GO:0019083 & Viral transcription & 67 & 2.214876 & $6.48 \mathrm{E}-24$ \\
\hline \multirow[t]{5}{*}{2} & GO:0006355 & Regulation of transcription, DNA-templated & 66 & $1.26 \mathrm{E}+01$ & $3.17 \mathrm{E}-04$ \\
\hline & GO:0006351 & Transcription, DNA-templated & 76 & $1.45 \mathrm{E}+01$ & 0.002697 \\
\hline & GO:0006366 & Transcription from RNA polymerase II promoter & 25 & $4.77 \mathrm{E}+00$ & 0.0108 \\
\hline & GO:0000122 & $\begin{array}{l}\text { Negative regulation of transcription from RNA } \\
\text { polymerase II promoter }\end{array}$ & 31 & $5.92 \mathrm{E}+00$ & 0.023932 \\
\hline & GO:0045944 & $\begin{array}{l}\text { Positive regulation of transcription from RNA } \\
\text { polymerase II promoter }\end{array}$ & 38 & $7.25 \mathrm{E}+00$ & 0.043971 \\
\hline \multirow[t]{5}{*}{3} & GO:0006405 & RNA export from nucleus & 9 & 1.86722 & $7.36 \mathrm{E}-05$ \\
\hline & GO:0000398 & mRNA splicing, via spliceosome & 17 & $3.53 \mathrm{E}+00$ & 2.00E-04 \\
\hline & GO:0006406 & mRNA export from nucleus & 11 & $2.28 \mathrm{E}+00$ & $2.43 \mathrm{E}-04$ \\
\hline & GO:0016032 & Viral process & 21 & $4.36 \mathrm{E}+00$ & $3.31 \mathrm{E}-04$ \\
\hline & GO:0051301 & Cell division & 21 & $4.36 \mathrm{E}+00$ & 6.34E-04 \\
\hline \multirow[t]{5}{*}{4} & GO:0000398 & mRNA splicing, via spliceosome & 15 & $3.36 \mathrm{E}+00$ & $6.48 \mathrm{E}-04$ \\
\hline & GO:0006886 & Intracellular protein transport & 14 & $3.14 \mathrm{E}+00$ & $3.30 \mathrm{E}-03$ \\
\hline & GO:0006099 & Tricarboxylic acid cycle & 5 & $1.12 \mathrm{E}+00$ & 0.004148 \\
\hline & GO:0000184 & $\begin{array}{l}\text { Nuclear-transcribed mRNA catabolic process, } \\
\text { nonsense-mediated decay }\end{array}$ & 9 & $2.02 \mathrm{E}+00$ & 0.006867 \\
\hline & GO:0006541 & Glutamine metabolic process & 4 & 8.97E-01 & 0.010284 \\
\hline \multirow[t]{5}{*}{5} & GO:0006357 & $\begin{array}{l}\text { Regulation of transcription from RNA } \\
\text { polymerase II promoter }\end{array}$ & 12 & $5.77 \mathrm{E}+00$ & 0.00501 \\
\hline & GO:0045494 & Photoreceptor cell maintenance & 4 & $1.92 \mathrm{E}+00$ & 0.005265 \\
\hline & GO:0045664 & Regulation of neuron differentiation & 3 & $1.44 \mathrm{E}+00$ & 0.018871 \\
\hline & GO:0006351 & Transcription, DNA-templated & 29 & $1.39 \mathrm{E}+01$ & 0.036089 \\
\hline & GO:0045893 & Positive regulation of transcription, DNA-templated & 11 & $5.29 \mathrm{E}+00$ & 0.03745 \\
\hline \multirow[t]{5}{*}{6} & GO:0008380 & RNA splicing & 11 & $6.36 \mathrm{E}+00$ & 2.89E-06 \\
\hline & GO:0006406 & mRNA export from nucleus & 9 & $5.20 \mathrm{E}+00$ & $3.46 \mathrm{E}-06$ \\
\hline & GO:0075733 & Intracellular transport of virus & 6 & $3.47 \mathrm{E}+00$ & $9.57 \mathrm{E}-05$ \\
\hline & GO:0006409 & tRNA export from nucleus & 5 & $2.89 \mathrm{E}+00$ & $1.91 \mathrm{E}-04$ \\
\hline & GO:0010827 & Regulation of glucose transport & 5 & $2.89 \mathrm{E}+00$ & $2.15 \mathrm{E}-04$ \\
\hline \multirow[t]{3}{*}{7} & GO:0060337 & Type I interferon signaling pathway & 16 & $2.19 \mathrm{E}+01$ & $5.44 \mathrm{E}-23$ \\
\hline & GO:0060333 & Interferon- $\gamma$-mediated signaling pathway & 12 & $1.64 \mathrm{E}+01$ & $6.13 \mathrm{E}-15$ \\
\hline & GO:0006955 & Immune response & 17 & $2.33 \mathrm{E}+01$ & $1.28 \mathrm{E}-11$ \\
\hline
\end{tabular}

GO, Gene Ontology; SRP, signal recognition particle; tRNA, transfer RNA.

The GO functional enrichment analysis revealed that module 2 was mostly enriched in transcriptional regulatory processes, including the regulation of DNA-templated transcription and transcription from the RNA polymerase II promoter. Module 2 was mostly enriched in KEGG pathways associated with metabolic processes, including the notch signaling pathway, the RNA degradation pathway, pathways in cancer and ubiquitin mediated proteolysis. Since the occurrence and development of cancer is accompanied by the abnormal expression of oncogenes or anti-oncogenes, the enriched pathways that module 2 is involved in may regulate the translation of protein-coding genes involved in the cell cycle or cell proliferation. Therefore, module 2 may be the most critical in the occurrence and development of UM.

The present study examined the degree of connectivity between critical genes to determine the interactions between 
Table II. KEGG pathway enrichment analysis of genes in the co-expression modules.

\begin{tabular}{|c|c|c|c|c|c|}
\hline Module & KEGG no. & Pathway & No. of genes & Background genes (\%) & P-value \\
\hline \multirow[t]{5}{*}{1} & hsa03010 & Ribosome & 82 & 2.710744 & $6.68 \mathrm{E}-26$ \\
\hline & hsa00190 & Oxidative phosphorylation & 63 & 2.082645 & 3.34E-13 \\
\hline & hsa05016 & Huntington's disease & 71 & 2.347107 & 8.71E-09 \\
\hline & hsa05012 & Parkinson's disease & 57 & 1.884298 & $1.14 \mathrm{E}-08$ \\
\hline & hsa05010 & Alzheimer's disease & 64 & 2.115702 & $1.41 \mathrm{E}-08$ \\
\hline \multirow[t]{6}{*}{2} & hsa04330 & Notch signaling pathway & 7 & 1.335878 & 2.37E-03 \\
\hline & hsa03018 & RNA degradation & 7 & 1.335878 & 2.31E-02 \\
\hline & hsa05200 & Pathways in cancer & 19 & 3.625954 & 3.37E-02 \\
\hline & hsa04120 & Ubiquitin mediated proteolysis & 9 & 1.717557 & 4.37E-02 \\
\hline & hsa04141 & Protein processing in endoplasmic reticulum & 10 & 1.908397 & $5.47 \mathrm{E}-02$ \\
\hline & hsa05169 & Epstein-Barr virus infection & 10 & 1.908397 & $9.76 \mathrm{E}-02$ \\
\hline \multirow[t]{5}{*}{3} & hsa04120 & Ubiquitin mediated proteolysis & 16 & 3.319502 & 2.50E-06 \\
\hline & hsa03040 & Spliceosome & 14 & 2.904564 & 4.08E-05 \\
\hline & hsa03018 & RNA degradation & 9 & 1.86722 & 8.54E-04 \\
\hline & hsa03015 & mRNA surveillance pathway & 8 & 1.659751 & $9.59 \mathrm{E}-03$ \\
\hline & hsa03013 & RNA transport & 11 & 2.282158 & $1.46 \mathrm{E}-02$ \\
\hline \multirow[t]{5}{*}{4} & hsa04120 & Ubiquitin mediated proteolysis & 11 & 2.466368 & 2.30E-03 \\
\hline & hsa01100 & Metabolic pathways & 47 & 10.53812 & 2.54E-03 \\
\hline & hsa00020 & Citrate cycle (TCA cycle) & 5 & 1.121076 & 6.38E-03 \\
\hline & hsa01200 & Carbon metabolism & 8 & 1.793722 & 2.35E-02 \\
\hline & hsa03015 & mRNA surveillance pathway & 7 & 1.569507 & $2.68 \mathrm{E}-02$ \\
\hline \multirow[t]{3}{*}{6} & hsa03013 & RNA transport & 7 & 4.046243 & 0.002524 \\
\hline & hsa03040 & Spliceosome & 5 & 2.890173 & 0.022281 \\
\hline & hsa05202 & Transcriptional misregulation in cancer & 5 & 2.890173 & 0.046592 \\
\hline \multirow[t]{5}{*}{7} & hsa04612 & Antigen processing and presentation & 12 & 16.43836 & $1.77 \mathrm{E}-13$ \\
\hline & hsa05168 & Herpes simplex infection & 14 & 19.17808 & $9.17 \mathrm{E}-12$ \\
\hline & hsa05150 & Staphylococcus aureus infection & 8 & 10.9589 & $1.51 \mathrm{E}-08$ \\
\hline & hsa05332 & Graft-vs.-host disease & 7 & 9.589041 & $2.14 \mathrm{E}-08$ \\
\hline & hsa05416 & Viral myocarditis & 8 & 10.9589 & 2.23E-08 \\
\hline
\end{tabular}

KEGG, Kyoto Encyclopedia of Genes and Genomes; TCA, tricarboxylic acid.

the co-expression modules. The results demonstrated that there was a significant difference in the interactions among different modules; this may be as a result of their different functions. The results identified that there was strong interaction connectivity between modules 2 and 3 and modules 3 and 6. It was also revealed that ubiquitin mediated proteolysis was significantly enriched in modules 2-4. Several studies have reported that ubiquitination is associated with the occurrence and development of cancer when accompanied by the dysregulation of oncogenes $(15,16)$. Mutation of the cellular tumor antigen p53 gene has been reported to be associated with the occurrence of the majority of different types of cancer. The ubiquitination pathway regulates p53 tumor suppressor protein stability, localization, and functions in normal and cancerous cells (17). Therefore, it has been suggested that ubiquitination serves an essential role in the occurrence and development of UM, as it was the most enriched function according to the functional enrichment analysis, and it can disturb the expression of relevant proteins involved in the cell cycle and proliferation. Ubiquitination is an important post-translational protein modification that regulates a host of critical cellular processes. It has been previously reported that the inhibition of ubiquitination was very effective in the treatment of multiple myeloma (18). Proteasome-mediated degradation is a common mechanism by which cells renew their intracellular proteins and maintain protein homeostasis (19). Cellular activity is often initiated by enzymes, which are proteins that are closely associated with cell cycle processes. The results of the present study suggest that ubiquitin is able to regulate the cell system within UM by controlling the activity or degradation of the majority of proteins in the cells of the eye. Since the majority of patients with UM succumb to liver metastases (20), the reason for which is unclear, the enriched signaling pathway identified in the present study may help to clarify the metastasis of UM tumors $(21,22)$. The inhibition of the ubiquitin mediated proteolysis pathway or the genes included in the co-expression modules may be an effective treatment for UM (15). 
In summary, module 2 was regarded as the most critical module in the development of UM disease. The ubiquitin mediated proteolysis pathway was identified as being significantly enriched in three modules, meaning the constituent proteins could have potential as diagnostic and prognostic biomarkers of $\mathrm{UM}$; however, further research is required to investigate this.

\section{Acknowledgements}

Not applicable.

\section{Funding}

The current study was supported by the Funds for Guangxi Zhuang Autonomous Region Science And Technology Hall (grant no. 114000-3B-86).

\section{Availability of data and materials}

The analyzed data sets generated during the present study are available from the corresponding author on reasonable request.

\section{Authors' contributions}

QW and MY conceived and designed the study. XH, HY and DH performed the computational experiments. QW and MY wrote the paper. CJW and JL reviewed and edited the manuscript.

\section{Ethics approval and consent to participate}

Not applicable.

\section{Patient consent for publication}

Not applicable.

\section{Competing interests}

All authors declare that they have no competing interests.

\section{References}

1. Stang A, Parkin DM, Ferlay J and Jöckel KH: International uveal melanoma incidence trends in view of a decreasing proportion of morphological verification. Int J Cancer 114: 114-123, 2005.

2. Singh AD and Topham A: Incidence of uveal melanoma in the United States: 1973-1997. Ophthalmology 110: 956-961, 2003.

3. Egan KM, Seddon JM, Glynn RJ, Gragoudas ES and Albert DM: Epidemiologic aspects of uveal melanoma. Surv Ophthalmol 32: 239-251, 1988
4. Bedikian AY, Legha SS, Mavligit G, Carrasco CH, Khorana S, Plager C, Papadopoulos N and Benjamin RS: Treatment of uveal melanoma metastatic to the liver: A review of the M.D. Anderson Cancer Center experience and prognostic factors. Cancer 76: 1665-1670, 1995.

5. Clarke C, Madden SF, Doolan P, Aherne ST, Joyce H, O'Driscoll L, Gallagher WM, Hennessy BT, Moriarty M, Crown J, et al: Correlating transcriptional networks to breast cancer survival: A large-scale coexpression analysis. Carcinogenesis 34: 2300-2308, 2013.

6. Wan Q, Tang J, Han Y and Wang D: Co-expression modules construction by WGCNA and identify potential prognostic markers of uveal melanoma. Exp Eye Res 166: 13-20, 2018.

7. Langfelder $P$ and Horvath S: WGCNA: An R package for weighted correlation network analysis. BMC Bioinformatics 9: $559,2008$.

8. Shi Z, Derow CK and Zhang B: Co-expression module analysis reveals biological processes, genomic gain, and regulatory mechanisms associated with breast cancer progression. BMC Syst Biol 4: 74, 2010.

9. Udyavar AR, Hoeksema MD, Clark JE, Zou Y, Tang Z, Li Z, Li M, Chen H, Statnikov A, Shyr Y, et al: Co-expression network analysis identifies Spleen Tyrosine Kinase (SYK) as a candidate oncogenic driver in a subset of small-cell lung cancer. BMC Syst Biol 7 (Suppl 5): S1, 2013.

10. Ihaka R and Gentleman R: R: A language for data analysis and graphics. J Comput Graph Stat 5: 299-314, 1996.

11. Dennis G Jr, Sherman BT, Hosack DA, Yang J, Gao W, Lane HC and Lempicki RA: DAVID: Database for annotation, visualization, and integrated discovery. Genome Biol 4: P3, 2003.

12. Ashburner M, Ball CA, Blake JA, Botstein D, Butler H, Cherry JM, Davis AP, Dolinski K, Dwight SS, Eppig JT, et al: Gene ontology: Tool for the unification of biology. Nat Genet 25: 25-29, 2000.

13. Kanehisa M, Goto S, Kawashima S, Okuno Y and Hattori M: The KEGG resource for deciphering the genome. Nucleic Acids Res 32 (Database Issue): D277-D280, 2004.

14. Kanehisa M and Goto S: KEGG: Kyoto encyclopedia of genes and genomes. Nucleic Acids Res 28: 27-30, 2000.

15. Gong J, Cao J, Liu G and Huo JR: Function and mechanism of F-box proteins in gastric cancer (Review). Int J Oncol 47: 43-50, 2015.

16. Testa U: Proteasome inhibitors in cancer therapy. Curr Drug Targets 10: 968-981, 2009.

17. Sane S and Rezvani K: Essential roles of E3 ubiquitin ligases in p53 regulation. Int J Mol Sci 18: E442, 2017.

18. Weathington NM and Mallampalli RK: Emerging therapies targeting the ubiquitin proteasome system in cancer. J Clin Invest 124: 6-12, 2014

19. Liu J, Shaik S, Dai X, Wu Q, Zhou X, Wang Z and Wei W: Targeting the ubiquitin pathway for cancer treatment. Biochim Biophys Acta 1855: 50-60, 2015.

20. Gragoudas ES, Egan KM, Seddon JM, Glynn RJ, Walsh SM, Finn SM, Munzenrider JE and Spar MD: Survival of patents with metastases from uveal melanoma. Ophthalmology 98: 383-390, 1991.

21. Harbour JW, Onken MD, Roberson ED, Duan S, Cao L, Worley LA, Council ML, Matatall KA, Helms C and Bowcock AM: Frequent mutation of BAP1 in metastasizing uveal melanomas. Science 330: 1410-1413, 2010.

22. Woodman SE: Metastatic uveal melanoma: Biology and emerging treatments. Cancer J 18: 148-152, 2012

This work is licensed under a Creative Commons Attribution-NonCommercial-NoDerivatives 4.0 International (CC BY-NC-ND 4.0) License. 